\title{
Impacts of Covid-19 on Mental Health and Suicide Risk in Occupational Settings: Implications for Suicide Prevention and Research
}

Ella Arensman

Prof. Ella Arensman is Research Professor with the School of Public Health, University College Cork and Chief Scientist with the National Suicide Research Foundation (NSRF), Ireland. She is Vice President of the European Alliance Against Depression, and past President of the International Association for Suicide Prevention. She is also Visiting Professor with the Australian Institute for Suicide Research and Prevention, Griffith University, Brisbane. She has been involved in research and prevention of suicide, self-harm and related issues for more than 30 years, with emphasis on risk and protective factors associated with suicide and self-harm, and effectiveness of preventive programmes. In Ireland, she played a key role in developing the first and second National Suicide Prevention Programme: Reach Out, 2005-2014, and Connecting for Life, 2015-2020.

Abstract. The impacts of the Covid-19 pandemic are far reaching and long lasting for people in many work settings, and especially for those in Small and Medium Enterprises (SMEs) in terms of people's wellbeing and mental health and the social and economic impacts. A review of the impacts of Covid-19 and associated movement restriction measures on people with psychiatric disorders and non-clinical mental health difficulties in occupational settings has been conducted by the MINDUP (Mental Health Promotion and Intervention in Occupational Settings) consortium. Considering the short-term and long-term impacts of Covid-19, including staff stress and anxiety, reduced quality and perhaps intensity of prevention of and care for those with mental disorders, or other impacts associated with the Covid-19-crisis, the MINDUP project is timely while it offers to SMEs support and intervention tools with a specific focus on alleviating depression and anxiety among staff. The reaction to the Covid-19 pandemic led to several changes in occupational settings such as increased and changed emotional work demands for some professionals, e.g. health care workers; exposure to infected patients and remote working. While many large organizations usually support their staff with Employee Assistance Programmes or workplace health promotion 
to support their employees in times of crisis, this is commonly not the case in SMEs and therefore the impact of Covid-19 may be exacerbated in these settings. In addition, loss of employment and financial stressors are wellrecognised risk factors for suicide. Governments should therefore provide financial safety nets, and consideration must be given not only to individuals' current situations but also their futures. For example, many young people have had their education interrupted and are anxious about their prospects. Educational institutions must seek alternative ways. The MINDUP project began in January 2020 and aims to improve mental health and wellbeing in the workplace by developing, implementing and evaluating a multilevel intervention targeting both prevalent psychiatric disorders (depression, anxiety disorders) and non-clinical aspects of mental health (stress, burnout, wellbeing, depressive symptoms). In addition, evidence-based tools and interventions will presented that can be included in response plans, implemented in SMEs and other occupational settings. 